\title{
Synthesis and comparative study of thermal, electrochemical, and cytotoxicity properties of graphene flake and sheet
}

\begin{abstract}
Two types of graphene, namely few-layer flake and multilayer sheet, were produced by chemical vapor deposition on nickel catalyst at high temperature $\left(1050{ }^{\circ} \mathrm{C}\right)$ using different reaction times and cooling rates and their properties characterized and compared. The number of layers, morphology, structure, graphitization, composition, and surface area were studied using scanning electron microscopy, transmission electron microscopy, electron-dispersive X-ray analysis, Raman spectroscopy, and Brunauer-Emmett-Teller (BET) surface area measurements, respectively. Further properties of these nanomaterials, including their thermal stability, electrochemical properties, and cytotoxicity, were also comprehensively investigated.
\end{abstract}

Keyword: Graphene flake; Graphene sheet; Chemical vapor deposition; Thermal and electrical properties; Cytotoxicity effects 